\title{
Genetic diversity and pathogenicity of Blastocystis
}

\author{
Bogumiła Skotarczak ${ }^{1, A-F}$ \\ Department of Genetics, Faculty of Biology, University of Szczecin, Poland \\ A - Research concept and design, B - Collection and/or assembly of data, C - Data analysis and interpretation, \\ $D$ - Writing the article, E - Critical revision of the article, F - Final approval of article
}

Skotarczak B. Genetic diversity and pathogenicity of Blastocystis. Ann Agric Environ Med. 2018; 25(3): 411-416. doi: 10.26444/aaem/81315

\begin{abstract}
Blastocystis is a unicellular, anaerobic protist which lives in the intestinal tract of diverse animals, including humans. It was found that the host specificity and the pathogenic potential of different isolates are correlated with sequence variations in the SSU-rRNA gene. Identification of the organism to the species level is still an unresolved challenge. Genetic diversity revisions have led to the identification of 17 subtypes (STs) within the Blastocystis genus, and 9 (ST1 to ST9) have been reported in humans with varying prevalence. Since the members of the genus revealed a large genetic diversity, several molecular modalities of subtyping methods have been developed. Numerous studies on conveying the pathogenic potential to the molecular subtypes are available, but they could not be compared or analysed with the different molecular techniques employed. The use of different approaches may give false positives during diagnosis and the possibility of missed infections. A review of recent scientific literature indicates that the development of PCR assays is needed for molecular epidemiology and for mixed infections in health and disease cohorts, and also to help identify sources of Blastocystis transmission to humans, as well as to identify potential animal and environmental reservoirs. This review summarizes some of the recent progress and improvements in Blastocystis research on genetic diversity, taxonomy, molecular epidemiology, pathogenicity and subtyping methods.
\end{abstract}

\section{Key words}

Blastocystis, taxonomy, pathogenicity, molecular epidemiology, subtyping methods

\section{INTRODUCTION}

Blastocystis sp. is an intestinal parasite with worldwide distribution, colonizing humans and a wide range of animals $[1,2]$. Blastocystis is a common unicellular anaerobic eukaryote that inhabits the large intestine and finding it in the faeces of mammals and birds suggests its zoonotic potential and that these animals may be the source of many human infections [3]. Blastocystis has been shown to be transmitted through the faecal-oral route, for example, through contaminated water [4]. The prevalence of Blastocystis spp. is reported in many parasite assessments performed throughout the world. Published human infection rates fall anywhere between 1 $60 \%$ or even to $100 \%$, depending on geographic distributions, economic status, in developed or in developing countries, etc. Blastocystis colonisations may concern more than 1 billion people globally $[5,6]$, although it is currently not possible to distinguish between colonisation and infection. At present, its clinical and public health significance remains unclear, mainly because it is common in both healthy people and patients suffering from intestinal symptoms, including common manifestations such as diarrhoea and irritable bowel syndrome $[7,8]$.

The diagnostic technique used for examining the infection rates is another important variability that can influence the real prevalence [9]. For many years, microscopic examination has been the only tool available for detection of gastrointestinal parasites in stool samples in routine diagnostic laboratories. Today, molecular methods through

Address for correspondence: Bogumiła Skotarczak Department of Genetics, Faculty of Biology, University of Szczecin, Felczaka 3c, 71-412 Szczecin, Poland E-mail: boskot@univ.szczecin.pl; genetyka@univ.szczecin.pl

Received: 04.10.2017; accepted: 19.12.2017; first published: 23.01.2018
DNA detection of parasite give several advantages, such as an increased sensitivity and specificity, and the possibility for molecular typing.

\section{OBJECTIVE}

The main aim of this review is evaluation of the recent information on the pathogenicity, prevalence and genetic diversity of Blastocystis sp. Additionally, the application and suitability of subtyping methods is discussed.

Taxonomy. The identification of the organism at the species level is still an unresolved challenge. First, Blastocystis was originally named $B$. hominis, but recent phylogenetic studies indicate limiting the name to Blastocystis species, because of genetic diversity revealed with the members within the genus [10]. Earlier, the species name was made based on the host from which was isolated such as $B$. hominis (from humans), $B$. ratti (from rats). It was then found that the host specificity and the pathogenic potential of different isolates are correlated with sequence variations in the SSU-rRNA gene [11]. Based on these variations, the members of the genus are ordered into several subtypes (STs), which could be named as species [12]. Later, it was discovered that humans were host to a number of diverse, small subunit rRNA gene (SSU-rDNA)-based subtypes of Blastocystis, and that most of these subtypes also originated from other mammalian or avian hosts. This predestined that the host-linked binomial species names were invalid, as the same organism was being named by multiple terms [13]. For example, one grouping of Blastocystis hominis proved to be genetically indistinguishable from $B$. ratti; both are now known as Blastocystis subtype 4 (ST4). 
For this reason, currently it is proper to use the nomenclature of the organism as "Blastocystis species" followed by the ST number, and it must be additionally characterized only by molecular subtyping of the SSU-rRNA.

Molecular epidemiology. Could some human infections result from zoonotic transmission? Blastocystis sp., as noted above, is a common intestinal protist occurring in humans and in a wide range of animals worldwide. A wide genetic diversity of this parasite has been demonstrated and 17 subtypes (STs) have already been identified in mammalian and avian hosts [3]. Among the 17 mammalian and avian STs, 9 (ST1 to ST9) have been reported in humans with varying prevalence $[3,9]$ and most frequently $(90 \%)$ belonged to ST1-ST4, with a predominance of ST3 (around 60\% of these isolates). Among these subtypes, ST1, 2 and 4 have been described as having a low host specificity and are probably zoonotic infections [3]. ST1 and ST2 are found in humans and a wide range of animals, including monkeys, cattle, chickens, pigs, dogs, and non-human primates [14-17]. ST3 has been classified as more anthroponotic and this subtype has also been found in non-human primates, pigs and cattle $[17,18]$. ST4 has been described in rodents and monkeys $[14,17,18]$. Moreover, the 4 most common STs in humans have also been identified in other hosts, the most frequent of which were other primates, but they have also been found in numerous hoofed mammals, rodents and birds $[13,19]$. On the contrary, the rarer subtypes in humans (STs 5-8) were also more commonly discovered in other hosts: ST5 is common in livestock, apes, and old world monkeys, but rare in humans $[3,9,18]$, while ST6 and ST7 are mostly found in birds, and ST6 has also been described in livestock and humans, while ST7 has also been found in humans [3, 20-22]. ST8 has been recognized in marsupials, different species of captive primates, their caregivers, and in pheasant $[18,19,22]$, and ST9 has been reported in humans $[15,22]$. It has been suggested that these rarer subtypes in humans are of zoonotic derivation, and there is some confirmation to support that ST8 has frequently been found in zookeepers who work with non-human primates [19].

The phylogenetic analysis by Alfellani et al. [3] discovered that human isolates create a separate subgroup within this subtype distinct from the animal isolates. With the exception of ST9 only found in humans, the other 8 STs show low to moderate host specificity also by inhabiting various animal groups [3, 23, 24]. Many authors include these animal groups as representatives of potential reservoirs of zoonotic transmission $[16,25]$. One more confirmation for the zoonotic potential of Blastocystis sp., is the high occurrence of this parasite among people handling animals, compared with those without contact with animals $[18,20,26]$. The possibility of transmission of the parasite between human and animal hosts has been demonstrated by experimental infections of chickens and rats with human isolates $[27,28]$.

Since several STs are common to humans and animals, it has been suggested that a percentage of human infections may be a consequence of zoonotic transmission, but the contribution of each animal source to human infection should be clarified [29]. A new study by Cian et al. [29] is attempting to expand knowledge about the epidemiology and host specificity of this parasite with the investigation concentrating on animal groups in conjunction with a number of species screened. A total of 307 stool samples from 161 mammalian and non-mammalian species in 2 French zoos were selected by real-time PCR for the presence of Blastocystis sp. More than $32.0 \%$ of the animal samples and almost $38.0 \%$ of the species tested were shown to be infected with the parasite. A total of 111 animal Blastocystis sp. isolates were subtyped and 11 of the 17 mammalian and avian STs, as well as supplementary STs previously recognized in reptiles and insects were found with a changing prevalence, according to animal groups. These data were combined with those obtained from previous examinations to estimate the potential risk of zoonotic transmission of Blastocystis sp. through the comparison of ST distribution between human and animal hosts. By combining obtained molecular data with data obtained in previous examinations, and comparing the summarized overall ST distribution between animals and humans, it appears that non-human primates, artiodactyls, especially livestock and birds, may serve as reservoirs for human infection, particularly in animal handlers. However, other mammals, such as carnivores, and non-mammalian groups like reptiles or insects, do not seem to indicate that they are sources of Blastocystis sp. infection in humans. The authors suggest that more epidemiological data is needed to completely identify the potential animal reservoirs of human infection and add to our understanding of the circulation of Blastocystis sp. in animal and human populations.

Pathogenicity of Blastocystis sp. Is a relationship possible in which Blastocystis does not have a pathogenic role in animals? It seems that there are no consequences in animals infected with Blastocystis and all animals appear to be healthy with no observed symptoms of disease. This is quite different from human infections where there are huge symptominfection rates [30-32]. It shows the possible relationship where Blastocystis does not have a pathogenic role in animals. Blastocystis was shown to be transmitted through the faecaloral route, as well as through contaminated water [4]. It is possible to say that the faecal-oral route may be the main route of transmission in the community-based groups of animals, such as the apes and elephants. The ape communities all eat and defecate in the same areas in their enclosures as well as play together and groom each other. If one animal harboured Blastocystis, it would be easy for that animal to spread it among the rest of the group. The Meerkat group were the only animals that live in a large community in the zoo that did not harbour Blastocystis. All the other community living animals were almost all positive for Blastocystis. This shows the easy transmission of this parasite in a group and how simply it can be to spread through the faecal oral route.

Most animal studies were carried out on domestic animals and animals in zoos. It would be interesting to compare the results of the zoo animals with their wild relatives to determine if the same subtypes and infection rates are found. It is not possible to describe a true representation of Blastocystis infection rates and subtypes present in animals if only zoo animals or domestic animals have been studied and only from specific areas of the world.

Human pathogenicity of Blastocystis sp. remains controversial and it has been suggested that it may be associated with certain subtypes of organism. Since $B$. hominis can be found in both symptomatic and asymptomatic patients (where its living may be commensal without any pathogenic effect), there are disputatious opinions about its 
pathogenicity [33-35]. A very high prevalence of Blastocystis sp. obviously invokes the question of the influence of this parasite on human health; however its role in human health and disease is uncertain [36,37]. Genomic data by Denoeud et al. [38] combined with in-vitro and in-vivo studies of $\mathrm{Wu}$ et al. [39] and Ajjampur et al. [28] allowed the identification of recognized virulence factors and revealed the harmful influences of the parasite on the intestinal barrier, leading to credible models of pathogenesis [40]. Several papers also suggest that this parasite should be related with non-specific gastrointestinal symptoms like diarrhoea, abdominal pain, and vomiting, and additionally, an association between B. hominis and irritable bowel syndrome (IBS) [40] and urticarial [41] is suggested.

Based on gene analysis of small-subunit ribosomal RNA (SSU-rRNA), as mentioned above, Blastocystis sp. isolates were finally classified into subtypes (ST1-ST17), which colonize a wide range of hosts, including humans and animals, both mammalian and non-mammalian $[10,42]$. Nine of 17 STs have been identified in humans, and one recent working hypotheses is that differences in clinical consequence may depend on differences in subtypes. In 1997, Clark suggested that different subtypes with different pathological potentials may exist [43], and Kaneda et al. [44] proposed that STs 1, 2, and 4 might be answerable for gastrointestinal symptoms. In 2012, Poirier et al. [40] designated that ST7 is connected with IBS, and Wawrzyniak et al. [45] discovered that ST7 probably uses hydrolases to destroy host tissues. What is more, the occurrence of gut microbiota appears to be important for the pathogenic countenance of Blastocystis [46]. Later, 9 of 17 subtypes of Blastocystis have been reported in humans and some STs such as ST10 and ST14 are present only in cattle, never in humans, even after direct contact [3]. Some STs show host specificity with variable geographic distribution. In America, there is a high prevalence of ST1 and ST2, in Australia, Europe, and South Eastern Asia - ST1 and ST3, and in Europe - ST4 [10]. A recent study from SouthAmerica showed the presence of ST4 in humans [47] and latter STs 1, 2 and 3 were detected as the most frequent [48]. People are colonized mainly by ST1 through ST4, containing over $90 \%$ of assessments; but depending on the regions and countries, infection by ST5 through ST9 is also detected $[10,20]$. Nevertheless, that ST3 is the most common ST in humans worldwide, and its occurrence is a frequent finding in analyses of ST distribution, regardless of the geographic derivation of the population $[18,25,49,50]$.

In contrast, some studies have presented no distinct differences in STs between isolates from symptomatic and asymptomatic groups with gastrointestinal symptoms [15]. In numerous other investigations there was no association between Blastocystis subtypes and gastrointestinal symptoms $[31,51,52]$. They identified ST3 as the most frequent subtype in both groups (symptomatic and asymptomatic), but did not observe a significant relationship between this subtype and gastrointestinal symptoms. Recently, it has been also investigated the connection between the symptomatology and subtypes of Blastocysis by Dogan et al. [53], but no statistically significant difference was found between the symptomatic and asymptomatic children groups in terms of the Blastocystis positivity and the distribution of subtypes.

Molecular identification and subtype analysis. It is proposed that diverse subtypes have different hosts, geographical distribution and ways of transmission. Therefore, the subtyping of Blastocystis sp. is important for epidemiological studies because it helps to recognize routes of transmission and potential sources of a specific ST in a precise area. This new information can help to broaden knowledge about the pathogenicity of Blastocystis sp. [54].

Microscopic checking of stool samples for the detection of cysts, oocysts, and trophozoites continues to be the choice of the diagnostic method for many laboratories [55]; however, the method requires technical expertise, and it is laborious; it can also be insensitive when infection is on low levels. Currently, molecular methods, including polymerase chain reaction (PCR), are available for detecting and identifying intestinal parasites of protists, and demonstrate high sensitivity and specificity with respect to conventional methods such as microscopy $[56,57]$.

Although after more than a dozen years, molecular analyses of Blastosystis is a leading method worldwide, there is still no consensus for the best method to use for STs. Numerous studies on the transmission of pathogenic potential to the molecular subtypes are available, but they cannot be compared or analysed with the different molecular techniques employed [6, 12]. Among methods for Blastocystis STs, there are examples of significantly different results from the same country, dependently of the methodology used [12].

Generally, 2 distinct approaches of analysis are employed; first, with small subunit rRNA gene (SSU-rDNA) PCR amplicons sequencing, and second, with direct assignment using diagnostic subtype-specific sequence-tagged sites (STS) primers for PCR [15]. The second method eliminates DNA sequencing, which is an advantage because DNA sequencing is not easily available to every laboratory. This approach involves the use of 7 PCR reactions, one for each of the subtypes 1-7, and should be regarded as involving a diagnostic method for each of these subtypes, avoiding the need for sequencing.

The first method is one of the most used approaches, which involves single-round PCR amplification and sequencing of partial SSU-rRNA gene of the parasites as the 'barcode' region. In recent times, a publicly available online facility was developed for fast and standardized identification of subtypes (ribosomal lineages) and subtype alleles (variation within subtypes), based on sequence data acquired by barcoding PCR [58]. Furthermore, a modified barcoding method is now available using nested PCR, which allows detecting mixed subtype infections $[6,58]$. 'DNA barcoding' is the term used to describe a method proposed for constructing a unique identifier for all living species [59]; Scicluna et al. [21] investigated the barcoding region for Blastocystis. A comparison of the STS method and barcoding indicated that barcoding should be preferred for a variety of motives. First and principal, barcoding allows detecting subtypes beyond STs 1-7 and further examination of genetic diversity. The barcode region has also been validated as a marker of overall genetic diversity of Blastocystis [60]. Moreover, using STS method, only known STs can be detected and, neither the specificity of STS has been identified nor the range of detectable STs extended. STS is certainly imperfect use if nonhuman samples are of interest $[6,13]$. STS typing is also more dependent on interpretation - size and specificity of bands, for example, in the case of sequence analysis. However, the study by Roberts et al. [61] highlighted the need for specific Blastocystis PCR methods to be advanced in order to stop 
the occurrence of false positives during diagnosis and the possibility of missed infections. In that study, targeting the SSU-rDNA specific for the Blastocystis, there were several specimens that were identified as positive by the PCR method, but after sequence analysis it was concluded that these products actually were derived from fungal species, in particular Mucor spp. and Rhizomucor spp. or from Cryptosporidium sp. The ability of the PCR and primers used in this study to cross-react with species other than Blastocystis indicates the necessity for sequence analysis to be performed on all PCR positive samples to confirm the identity of the PCR sequence. This also explains the difficulty in projecting primer pairs specific to Blastocystis. Molecular analysis of Blastocystis continuously results in new subtypes being identified as more animals and humans from different geographical sites are examined. This gives the choice of the right PCR technique and primer pairs essential for the probability to detect all new subtypes.

Mixed infection. Scanlan et al. [6] underlined that although most individuals seem to host only a single Blastocystis ST, many individuals host multiple Blastocystis STs, demonstrating that the occurrence of Blastocystis mixed-ST infections is much greater than previously thought, primarily due to methodological limitations. It is widely thought that there are difficulties in evaluating precisely the distribution of mixed infections due to the methodologies employed $[3,62]$. Dogan et al. [53] used real-time PCR for subtyping Blastocystis and mixed subtyping occurrence was found. Subtyping was successfully performed using sequencedtagged site (STS) primers and PCR in faecal samples of children with or without symptoms. The most frequently detected subtype was ST3 followed by ST1, ST4 and ST2 (8.7\%). The mixed subtypes were identified as ST1 + ST3, ST1 + ST2 and ST2 + ST3, and the authors concluded that this method is practical. Also, Dağc1 et al. [63] found the sensitivity and specificity of a real-time PCR array as the ability of multiple different STs to co-colonize a single host. Scanlan et al. [6] developed and applied Blastocystis STspecific nested PCR assay for investigation the most common subtypes of Blastocystis (ST1 - ST4) to a healthy human cohort $(n=50)$ and detected mixed infections in $22 \%$ of the cases, all of which had been identified as single-ST infections in a previous study. The obtained results show that certain STs are predominant as either single (ST3 and 4) or occur mixed (ST1), and comparative analyses with other primers used extensively previously $[64,65]$ for STspecific analysis, found them unsuitable for detection of mixed-and, in some cases, single-ST infections. The authors underline that the development of these nested PCR assays will facilitate future work on molecular epidemiology, and the significance of mixed infections in healthy and disease cohorts. Additionally, it will help in the recognition of sources of Blastocystis transmission to humans, including identifying potential animal and environmental reservoirs.

\section{CONCLUSIONS}

Although, a large number of molecular techniques have been developed for the identification of STs, a standard methodology has not yet been confirmed. The techniques commonly employed to identify human Blastocystis STs are PCR amplification with known sequence-tagged site (STS) primers or sequencing of the SSU rDNA - barcode region, followed by a search with known STs. But STS primers are limited to STs $1-7$, and the second method requires sequencing for subtyping, which is not available in all laboratories and is still a relatively laborious and expensive procedure. While STS primers can be detected in mixed STs, ST-identification with conventional direct sequencing of partial SSU rDNA cannot be performed in mixed-ST infections, although mixed-ST infections have been reported in human samples in various countries. The limits of first method seem to have been resolved by last study of Yoshikawa and Iwamasa [66] who developed ST-specific primers for the subtyping of human Blastocystis isolates, based on the unique nucleotide sequences of the SSU rRNA gene of 9 STs, all known as human STs.

This review of recent scientific literature indicates that the development of PCR assays is needed for molecular epidemiology and for mixed infections in both healthy and diseased cohorts, and also to help identify the sources of Blastocystis transmission to humans, as well as identifying potential animal and environmental reservoirs.

\section{REFERENCES}

1. Menounos PG, Spanakos G, Tegos N, Vassalos CM, Papadopoulou C, Vakalis NC. Direct detection of Blastocystis sp. in human faecal samples and subtype assignment using single strand conformational polymorphism and sequencing. Mol Cell Probes. 2008; 22(1): 24-29.

2. Alfellani MA, Stensvold CR, Vidal-Lapiedra A, Onuoha ES, FagbenroBeyioku AF, Clark CG. Variable geographic distribution of Blastocystis subtypes and its potential implications. Acta Trop. 2013a; 126: 11-18. doi:10.1016/j.actatropica.2012.12.011.

3. Alfellani MA, Taner-Mulla D, Jacob AS, Imeede CA, Yoshikawa $\mathrm{H}$, Stensvold CR, Clark CG. Genetic diversity of Blastocystis in livestock and zoo animals. Protist. 2013b; 164: 497-509. doi:10.1016/j. protis.2013.05.003.

4. Leelayoova S, Siripattanapipong S, Thathaisong U, Naaglor T, Taamasri P, Piyaraj P, Mungthin M. Drinking water: a possible source of Blastocystis spp. subtype 1 infection in schoolchildren of a rural community in central Thailand. Am J Trop Med Hyg. 2008; 79(3): 401-406.

5. El Safadi D, Gaayeb L, Meloni D, Cian A, Poirier P, Wawrzyniak I, Delbac F, Dabboussi F, Delhaes L, Seck M, Hamze M, Riveau G, Viscogliosi E. Children of Senegal River Basin show the highest prevalence of Blastocystis sp. ever observed worldwide. BMC Infect Dis. 2014; 14: 164. doi: 10.1186/1471-2334-14-164.

6. Scanlan PD, Stensvold CR, Cotter PD. Development and application of a Blastocystis subtype-specific PCR assay reveals that mixed-subtype infections are common in a healthy human population. Appl Environ Microbiol. 2015; 81(12): 4071-4076. doi: 10.1128/AEM.00520-15.

7. Scanlan PD, Stensvold CR, Rajilić-Stojanović M, Heilig HG, De Vos WM, O'Toole PW, Cotter PD. The microbial eukaryote Blastocystis is a prevalent and diverse member of the healthy human gut microbiota. FEMS Microbiol Ecol. 2014; 90: 326-330. doi: 10.1111/1574-6941.12396.

8. Krogsgaard LR, Engsbro AL, Stensvold CR, Nielsen HV, Bytzer P. The prevalence of intestinal parasites is not greater among individuals with irritable bowel syndrome: a population-based case-control study. Clin Gastroenterol Hepatol. 2015; 13(3): 507-513. doi: 10.1016/j. cgh.2014.07.065.

9. Clark CG, van der Giezen M, Alfellani MA, Stensvold CR. Recent developments in Blastocystis research. Adv Parasitol. 2013; 82: 1-32. doi: 10.1016/B978-0-12-407706-5.00001-0.

10. Stensvold CR, Suresh GK, Tan KS, Thompson RC, Traub RJ, Viscogliosi E, Yoshikawa H, Clark CG.. Terminology for Blastocystis subtypes - a consensus. Trends Parasitol. 2007; 23: 93-96. doi: 10.1016/j. pt.2007.01.004.

11. Noel C, Dufernez F, Gerbod D, Edgcomb VP, Delgado-Viscogliosi P, Ho LC, Singh M, Wintjens R, Sogin ML, Capron M, Pierce R, Zenner L, Viscogliosi E. Molecular phylogenies of Blastocystis isolates from 
different hosts: implications for genetic diversity, identification of species, and zoonosis. J Clin Microbiol. 2005; 43: 348-355. doi: 10.1128/ JCM.43.1.348-355.2005.

12. Stensvold CR. Blastocystis: genetic diversity and molecular methods for diagnosis and epidemiology. Trop Parasitol. 2013a; 3: 26-34. doi: 10.4103/2229-5070.113896.

13. Stensvold CR, Clark CG. Current status of Blastocystis: a personal view. Parasitol Int. 2016a; 65(6 Pt B): 763-771. doi: 10.1016/j. parint.2016.05.015.

14. Ramirez JD, Sánchez LV, Bautista DC, Corredor AF, Flórez AC, Stensvold CR. Blastocystis subtypes detected in humans and animals from Colombia. Infect Genet Evol. 2014; 22: 223-228. doi: 10.1016/j. meegid.2013.07.020.

15. Yoshikawa H, Wu Z, Kimata I, Iseki M, Ali IK, Hossain MB, Zaman V, Haque R, Takahashi Y. Polymerase chain reaction-based genotype classification among human Blastocystis hominis populations isolated from different countries. Parasitol Res. 2004; 92: 22-29. doi:10.1007/ s00436-003-0995-2.

16. Yoshikawa H, Wu Z, Pandey K, Pandey BD, Sherchand JB, Yanagi T, Kanbara H. Molecular characterization of Blastocystis isolates from children and rhesus monkeys in Kathmandu, Nepal. Vet Parasitol. 2009; 160: 295-300. doi:10.1016/j.vetpar.2008.11.029.

17. Rivera WL. Phylogenetic analysis of Blastocystis isolates from animal and human hosts in the Philippines. Vet Parasitol 2008; 156: 178-182. doi:10.1016/j.vetpar.2008.06.001.

18. Stensvold CR, Alfellani MA, Nørskov-Lauritsen S, Prip K, Victory EL, Maddox C, Nielsen HV, Clark CG. Subtype distribution of Blastocystis isolates from synanthropic and zoo animals and identification of a new subtype. Int J Parasitol. 2009; 39: 473-479. doi: 10.1016/j. ijpara.2008.07.006.

19. Alfellani MA, Jacob AS, Perea NO, Krecek RC, Taner-Mulla D, Verweij JJ, Levecke B, Tannich E., Clark CG, Stensvold CR. Diversity and distribution of Blastocystis sp. subtypes in non-human primates. Parasitol. 2013c; 140: 966-971. doi: 10.1017/S0031182013000255.

20. Parkar U, Traub RJ, Vitali S, Elliot A, Levecke B, Robertson I, Geurden T, Steele J, Drake B, Thompson RC. Molecular characterization of Blastocystis isolates from zoo animals and their animal-keepers. Vet Parasitol. 2010; 169: 8-17. doi:10.1016/j.vetpar.2009.12.032.

21. Scicluna SM, Tawari B, Clark CG. DNA barcoding of Blastocystis. Protist. 2006; 157(1): 77-85. doi:10.1016/j.protis.2005.12.001.

22. Petrasova J, Uzlíková M, Kostka M, Petrželková KJ, Huffman MA, Modrý D. Diversity and host specificity of Blastocystis in syntopic primates on Rubondo Island, Tanzania. Int J Parasitol. 2011; 41(11): 1113-1120. doi: 10.1016/j.ijpara.2011.06.010.

23. Santin M, Gomez-Munoz MT, Solano-Aguilar G, Fayer R. Development of a new PCR protocol to detect and subtype Blastocystis spp. from humans and animals. Parasitol Res. 2011; 109: 205-212. doi: 10.1007/ s00436-010-2244-9.

24. Yoshikawa H, Koyama Y, Tsuchiya E, Takami K. Blastocystis phylogeny among various isolates from humans to insects. Parasitol Int. 2016; 65(6 Pt B): 750-759. doi: 10.1016/j.parint.2016.04.004.

25. Nagel R, Cuttell L, Stensvold CR, Mills PC, Bielefeldt-Ohmann H, Traub RJ. Blastocystis subtypes in symptomatic and asymptomatic family members and pets and response to therapy. Intern Med J. 2012; 42: 1187-1195. doi: 10.1111/j.1445-5994.2011.02626.x.

26. Wang W, Owen H, Traub RJ, Cuttell L, Inpankaew T, BielefeldtOhmann H. Molecular epidemiology of Blastocystis in pigs and their in-contact humans in Southeast Queensland, Australia, and Cambodia. Vet Parasitol. 2014; 203: 264-269. doi: 10.1016/j. vetpar.2014.04.006

27. Iguchi A, Ebisu A, Nagata S, Saitou Y, Yoshikawa H, Iwatani S, et al. Infectivity of different genotypes of human Blastocystis hominis isolates in chickens and rats. Parasitol Int. 2007; 56: 107-112.

28. Ajjampur SSR, Png CW, Chia WN, Zhang Y, Tan KSW. Ex vivo and in vivo mice models to study Blastocystis spp. adhesion, colonization and pathology: closer to proving Koch's postulates. PLoS One 2016; 11: e0160458. doi: 10.1371/journal.pone.0160458.

29. Cian A, El Safadi D, Osman M, Moriniere R, Gantois N, BenamrouzVanneste S, Delgado-Viscogliosi P, Guyot K, Li L-L, Monchy S, Noël C, Poirier P, Nourrisson C, Wawrzyniak I, Delbac F, Bosc S, Chabé M, Petit T, Certad G, Viscogliosi E. Molecular epidemiology of Blastocystis sp. in various animal groups from two French zoos and evaluation of potential zoonotic risk. PLoS One 2017; 12(1): e0169659. doi: 10.1371/ journal.pone.0169659.

30. Yan Y, Su S, Lai R, Liao H, Ye J, Li X, Luo X, Chen G. Genetic variability of Blastocystis hominis isolates in China. Parasitol Res. 2006; 99: 597601. doi:10.1007/s00436-006-0186-z.
31. Ozyurt M, Kurt O, Mølbak K, Nielsen HV, Haznedaroglu T, Stensvold CR. Molecular epidemiology of Blastocystis infections in Turkey. Parasitol Int. 2008; 57(3): 300-306. doi: 10.1016/j.parint.2008.01.004.

32. Vogelberg C, Stensvold CR, Monecke S, Ditzen A, Stopsack K, HeinrichGräfe U, Pöhlmann C. Blastocystis sp. subtype 2 detection during recurrence of gastrointestinal and urticarial symptoms. Parasitol Int. 2010; 59: 469-471. doi:10.1016/j.parint.2010.03.009.

33. Tan KS. New insights on classification, identification, and clinical relevance of Blastocystis spp. Clin Microbiol Rev. 2008; 21: 639-665. doi:10.1128/CMR.00022-08.

34. Khademvatan S, Masjedizadeh R, Rahim F, Mahbodfar H, Salehi R, Yousefi-Razin E, Foroutan M. Blastocystis and irritable bowel syndrome: frequency and subtypes from Iranian patients Parasitol Int. 2017; 66: 142-145. doi: 10.1016/j.parint.2017.01.005.

35. Udkow MP, Markell EK. Blastocystis hominis: prevalence in asymptomatic versus symptomatic hosts J Infect Dis. 1993; 168: 242244.

36. Ajjampur SSR, Tan KSW. Pathogenic mechanisms in Blastocystis spp.Interpreting results from in vitro and in vivo studies. Parasitol Int. 2016; 65(6 Pt B): 772-779. doi: 10.1016/j.parint.2016.05.007.

37. Andersen LO, Stensvold CR. Blastocystis in health and disease: are we moving from a clinical to a public health perspective? J Clin Microbiol. 2016; 54: 524-528. doi: 10.1128/JCM.02520-15.

38. Denoeud F, Roussel M, Noel B, Wawrzyniak I, Da Silva C, Diogon M, Viscogliosi E, Brochier-Armanet C, Couloux A, Poulain J, Segurens B, Anthouard V, Texier C, Blot N, Poirier P, Ng GC, Tan KS, Artiguenave F, Jaillon O, Aury JM, Delbac F, Wincker P, Vivarès CP, El Alaoui H. Genome sequence of the stramenopile Blastocystis, a human anaerobic parasite. Genome Biol. 2011; 12(3): R29. doi: 10.1186/gb-2011-12-3-r29.

39. Wu Z, Mirza H, Tan KSW. Intra-subtype variation in enteroadhesion accounts for differences in epithelial barrier disruption and is associated with metronidazole resistance in Blastocystis subtype-7. PLoS Negl Trop Dis. 2014; 8: e2885 doi: 10.1371/journal.pntd.0002885.

40. Poirier P, Wawrzyniak I, Vivarès CP, Delbac F, El Alaoui H. New insights into Blastocystis spp.: a potential link with irritable bowel syndrome. PLoS Pathog. 2012; 8: e1002545. doi:10.1371/journal.ppat.1002545.

41. Lepczynska M, Chen WC, Dzika E. Mysterious chronic urticarial caused by Blastocystis spp.? Int J Dermatol. 2016; 55: 259-266. doi: 10.1111/ijd.13064

42. El Safadi D, Meloni D, Poirier P, Osman M, Cian A, Gaayeb L, Wawrzyniak I, Delbac F, El Alaoui H, Delhaes L, Dei-Cas E, Mallat H, Dabboussi F, Hamze M, Viscogliosi E. Molecular epidemiology of Blastocystis in Lebanon and correlation between subtype 1 and gastrointestinal symptoms. Am J Trop Med Hyg. 2013; 88: 1203-1206. doi: 10.4269/ajtmh.12-0777.

43. Clark CG. Extensive genetic diversity in Blastocystis hominis. Mol Biochem Parasitol. 1997; 87: 79-83. doi: 10.1016/S0166-6851(97)000467.

44. Kaneda Y, Horiki N, Cheng X, Tachibana H, Tsutsumi Y. Serologic response to Blastocystis hominis infection in asymptomatic individuals. Tokai J Exp Clin Med. 2000; 25: 51-56.

45. Wawrzyniak I, Poirier P, Viscogliosi E, Dionigia M, Texier C, Delbac F, Alaoui HE. Blastocystis, an unrecognized parasite: an overview of pathogenesis and diagnosis. Ther Adv Infect Dis 2013; 1: 167-178. doi:10.1177/2049936113504754.

46. Berrilli F, Di Cave D, Cavallero S, D'Amelio S. Interactions between parasites and microbial communities in the human gut. Front Cell Infect Microbiol. 2012; 2: 141. doi:10.3389/fcimb.2012.00141.

47. Ramirez JD, Sánchez A, Hernández C, Flórez C, Bernal MC, Giraldo JC, Reyes P, López MC, García L, Cooper PJ, Vicuña Y, Mongi F, Casero RD. Geographic distribution of human Blastocystis subtypes in South America. Infect Genet Evol. 2016; 41: 32-35. doi:10.1016/j. meegid.2016.03.017.

48. Ramirez JD, Flórez C, Olivera M, Bernal MC, Giraldo JC. Blastocystis subtyping and its association with intestinal parasites in children from different geographical regions of Colombia. PLoS One 2017; 12(2): e0172586. doi: 10.1371/journal.pone.0172586.

49. Forsell J, Granlund M, Stensvold CR, Clark CG, Evengård B. Subtype analysis of Blastocystis isolates in Swedish patients. Eur J Clin Microbiol Infect Dis. 2012; 31: 1689-1696. doi:10.1007/s10096-011-1416-6.

50. Meloni D, Sanciu G, Poirier P, El Alaoui H, Chabé M, Delhaes L, Dei-Cas E, Delbac F, Fiori PL, Di Cave D, Viscogliosi E. Molecular subtyping of Blastocystis sp. isolates from symptomatic patients in Italy. Parasitol Res. 2011; 109: 613-619. doi: 10.1007/s00436-011-2294-7.

51. Dogruman-Al F, Kustimur S, Yoshikawa H, Tuncer C, Simsek Z, Tanyuksel M, Araz E, Boorom K. Blastocystis subtypes in irritable bowel syndrome and inflammatory bowel disease in Ankara, Turkey. 
Mem Inst Oswaldo Cruz 2009; 104: 724-727. doi:10.1590/S007402762009000500011.

52. Jantermtor S, Pinlaor P, Sawadpanich K, Pinlaor S, Sangka A, Wilailuckana C, Wongsena W, Yoshikawa H. Subtype identification of Blastocystis spp. isolated from patients in a major hospital in northeastern Thailand. Parasitol Res. 2013; 112(4): 1781-1786. doi: 10.1007/s00436-012-3218-x.

53. Dogan N, Aydin M, Tuzemen NU, Dinleyici EC, Oguz I, DogrumanAl F. Subtype distribution of Blastocystis spp. isolated from children in Eskisehir, Turkey. Parasitol Int. 2017; 66(1): 948-951. doi: 10.1016/j. parint.2016.10.008.

54. Scanlan PD. Blastocystis past pitfalls and future perspectives. Trends Parasitol. 2012; 28: 327-334. doi: 10.1016/j.pt.2012.05.001.

55. McHardy IH, Wu M, Shimizu-Cohen R, Couturier MR, Humphries RM. Detection of intestinal protozoa in the clinical laboratory. J Clin Microbiol. 2014; 52:712-720. doi: 10.1128/JCM.02877-13.

56. Verweij JJ. Application of PCR-based methods for diagnosis of intestinal parasitic infections in the clinical laboratory. Parasitol. 2014; 141: 1863-72. doi: 10.1017/S0031182014000419.

57. Skotarczak B. Methods for parasitic protozoan detection in the environmental samples. Parasite 2009; 16(3): 183-190.

58. Stensvold CR, Clark CG. Molecular identification and subtype analysis of Blastocystis. Curr Protoc Microbiol. 2016b; 43: 20A.2.1-20A.2.10. doi: $10.1002 /$ cpmc.17.

59. Hebert PD, Cywinska A, Ball SL, de Waard J.R. Biological identifications through DNA barcodes. Proc R Soc London B. 2003; 270: 313-321.
60. Stensvold CR, Christiansen DB, Olsen KE, Nielsen HV. Blastocystis sp. subtype 4 is common in Danish Blastocystis-positive patients presenting with acute diarrhea. Am J Trop Med Hyg. 2011; 84: 883-885. doi:10.4269/ajtmh.2011.11-0005.

61. Roberts T., Stark D, Harkness J, Ellis J. Subtype distribution of Blastocystis isolates from a variety of animals from New South Wales, Australia. Veterinary Parasitol. 2013; 196: 85-89. doi: 10.1016/j. vetpar.2013.01.011.

62. Stensvold CR. Comparison of sequencing (barcode region) and sequence-tagged-site PCR for Blastocystis subtyping. J Clin Microbiol. 2013b; 51: 190-194. doi:10.1128/JCM.02541-12.

63. Dagci H, Kurt Ö, Demirel M, Mandiracioglu A, Aydemir S, Saz U, Bart A, VAN Gool T. Epidemiological and diagnostic features of Blastocystis infection in symptomatic patients in Izmir province, Turkey. Iran J Parasitol. 2014; 9(4): 519-529.

64. Lee LL, Chye TT, Karmacharya BM, Govind SK. Blastocystis sp.: waterborne zoonotic organisms, a possibility? Parasit Vec. 2012; 5: 130 doi: 10.1186/1756-3305-5-130.

65. Moosavi A, Haghighi A, Mojarad EN, Zayeri F, Alebouyeh M, Khazan $\mathrm{H}$, Kazemi B, Zali MR. Genetic variability of Blastocystis sp. isolated from symptomatic and asymptomatic individuals in Iran. Parasitol Res. 2012; 111: 2311-2315. doi:10.1007/s00436-012-3085-5.

66. Yoshikawa H, Iwamasa A. Human Blastocystis subtyping with subtype-specific primers developed from unique sequences of the SSU rRNA gene. Parasitol Int. 2016; 65(6 Pt B): 785-791. doi: 10.1016/j. parint.2016.03.002. 